Musées, Patrimoine et Culture scientifiques et techniques

$161 \mid 2015$

septembre-octobre 2015

\title{
«À la découverte du ciel », un outil pédagogique pour les nouvelles activités périscolaires
}

\section{Colette Cazin et Simon Lericque}

\section{OpenEdition \\ Journals}

Édition électronique

URL : http://journals.openedition.org/ocim/1567

DOI : $10.4000 /$ ocim. 1567

ISSN : 2108-646X

Éditeur

OCIM

Édition imprimée

Date de publication : 1 septembre 2015

Pagination : 25-29

ISSN : 0994-1908

Référence électronique

Colette Cazin et Simon Lericque, « «À la découverte du ciel », un outil pédagogique pour les nouvelles activités périscolaires ", La Lettre de l'OCIM [En ligne], 161 | 2015, mis en ligne le 01 septembre 2016, consulté le 30 avril 2019. URL : http://journals.openedition.org/ocim/1567 ; DOI : 10.4000/ocim.1567

Ce document a été généré automatiquement le 30 avril 2019.

Tous droits réservés 


\section{«À la découverte du ciel », un outil pédagogique pour les nouvelles activités périscolaires}

\section{Colette Cazin et Simon Lericque}

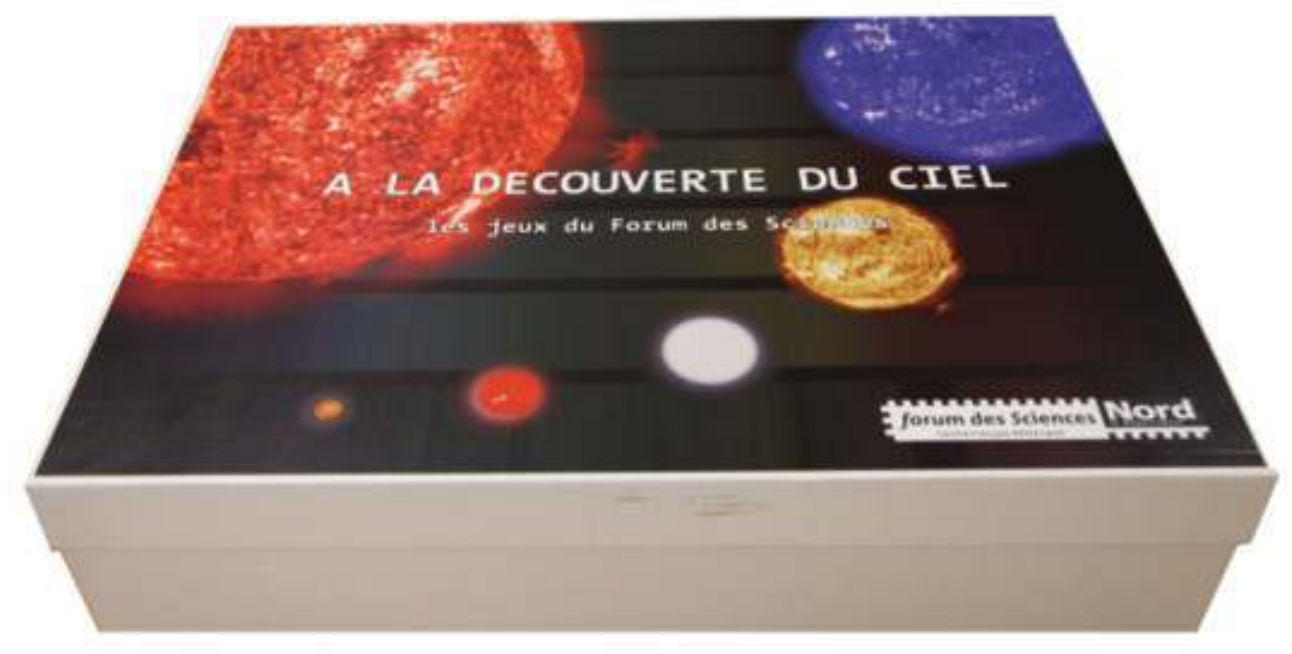

(C) Forum des Sciences/S. Lericque

Le Forum départemental des Sciences de Villeneuve-d'Ascq est un établissement spécialisé dans la diffusion de la culture scientifique, technique et industrielle. Il a pour mission de diffuser à un large public, notamment aux enfants et aux adolescents, les connaissances scientifiques et techniques, ainsi que de susciter l'intérêt des citoyens pour les enjeux de société liés à la science, à la recherche et à l'industrie. Le Forum qui fêtera en 2016 ses 20 ans compte $3000 \mathrm{~m}^{2}$ d'espaces ouverts au public, dont $1200 \mathrm{~m}^{2}$ d'espaces 
d'exposition. Il dispose des équipes dédiées aux expositions du « Petit Forum » pour les 3-6 ans, du plateau d'expositions, du Planétarium et des séances Énigma, et conçoit des outils itinérants pour faire découvrir la science dans la région Nord-Pas de Calais.

2 Depuis 2010, le Forum anime et fédère les initiatives menées autour de la science d'un réseau de 11 structures de culture scientifique en région Nord-Pas de Calais et en Belgique.

\section{Le Projet Scientifique et Culturel}

3 Le Forum départemental des Sciences crée, diffuse et promeut une culture scientifique à la fois accessible et ambitieuse. Son ambition est de permettre à chacun de devenir acteur d'un monde en pleine évolution. Pour cela, il faut sans cesse développer, échanger, transmettre de nouvelles clés de compréhension.

Il propose une programmation riche et sans cesse renouvelée pour répondre à la diversité des publics et de leurs attentes (groupes scolaires, de loisirs, adultes, enfants, familles, individuels...).

Le Projet Scientifique et Culturel adopté à l'unanimité par le Conseil général du Nord le 15 décembre 2014, a pour objectif central que « chacun puisse s'approprier la science pour redevenir acteur d'un monde en pleine évolution ».

6 Pour atteindre ces objectifs, le Forum départemental des Sciences s'appuie à la fois sur une vision humaniste des sciences et sur un savoir-faire reconnu en médiation culturelle. En effet, la médiation et l'animation sont au cœur des missions et activités du Forum et de ses agents. Il s'appuie simultanément sur un lieu culturel vivant et attractif et, pour toucher l'ensemble des citoyens sur les différents territoires, sur un réseau d'acteur (le réseau « Culture de Sciences en Nord-Pas de Calais ») qu'il anime. 




(c) Ville de Lille

\section{Le jeune public}

7 Le Forum s'adresse à tous les publics - enfants, jeunes et adultes, novices, amateurs et spécialistes, scolaires, familles et individuels - et construit une offre diverse construite avec le public. Dès son origine, il a particulièrement veillé à concevoir des outils, des activités en direction du jeune public. La création d'un espace spécifique pour les 3-6 ans en est une illustration parfaite. Par ailleurs, avant même la création de l'actuel Forum, l'association porteuse du projet concevait des outils itinérants à destination notamment du jeune public - mission qu'il poursuit encore aujourd'hui.

8 Le jeune public constitue l'essentiel des visiteurs du Forum, venu en groupe, sur le temps scolaire ou de loisirs, ou en famille. Les quelques 30 outils itinérants diffusés par le Forum sur le territoire national sont eux aussi à destination finale d'un jeune public circulant dans les écoles, les médiathèques, les centres sociaux ou culturels, les collectivités...

Ainsi les échanges avec le monde de l'éducation sont nombreux. Pour chaque conception, au moins un représentant de l'Éducation nationale collabore au sein des comités scientifiques de conception.

10 C'est donc fort d'une expérience de plus de 20 ans de médiation et de création d'outils jeune public que le Forum s'est lancé dans la création d'un outil spécifique pour les Nouvelles activités périscolaires (NAP) mises en place par les communes. 


\section{« À la découverte du ciel » : un outil d'animation adapté aux NAP}

11 À l'occasion de la mise en place des nouveaux temps d'animations périscolaires, le Forum départemental des Sciences a été sollicité à plusieurs reprises par diverses communes à la recherche d'animateurs ou d'activités. Les communes étant pour la plupart en recherche d'outils animés pour occuper les enfants sur un cycle de 6 à 10 séances d'environ une heure, les productions du Forum ne correspondaient pas à ce cahier des charges et la mise à disposition d'animateurs n'était pas possible. Le Forum a ensuite entrepris avec la Ville de Lille un travail sur mesure pour l'aider à concrétiser son projet de sciences. Après quelques temps d'échanges, le choix s'est finalement porté sur le thème de l'astronomie, sujet souvent apprécié par les élèves et parfois délaissé par les enseignants. Le service éducatif lillois souhaitait d'abord une intervention des médiateurs du Forum départemental des Sciences dans le cadre des NAP. Le Forum ne pouvait répondre à cette demande précise dans la mesure où ses effectifs en médiation étaient déjà restreints. Il a donc été décidé de s'orienter vers une solution basée sur la création d'une nouvelle forme d'outil itinérant complétée par une formation des animateurs lillois.

12 À l'époque, le catalogue de prestations itinérantes du Forum départemental des Sciences comportait plusieurs outils (une exposition panneaux cosmos, une histoire des représentations de l'Univers, une "Malle Cosmos", des planétariums mobiles sous dôme gonflable dotés de projecteurs numériques) mais ceux-ci n'étaient pas capables de répondre pleinement à la demande de la Ville de Lille.

13 Le développement d'un nouveau type de produit a donc été décidé. Plusieurs contraintes ont été fixées : il fallait un outil léger, compact, peu coûteux et permettant aux animateurs de mener 10 séances d'animations de $1 \mathrm{~h} 30$ pour une trentaine d'enfants. Le délai pour concevoir cette nouvelle boîte de jeux était aussi très court : quelques semaines à peine. Le tout était à livrer « clé en main » pour la rentrée scolaire de septembre 2014.

14 Afin de répondre au concept même des temps d'animations périscolaires - pouvant être en lien avec le programme de l'Éducation nationale mais sans être un cours magistral - le choix s'est porté sur le jeu. Ainsi, l'animation resterait pédagogique mais aussi ludique. Le choix a été fait dès le départ de « limiter » cette boîte de jeux aux élèves du cycle 3, les notions astronomiques abordées faisant écho au programme scolaire de ce niveau. Pour réaliser ce nouvel outil, l'équipe ayant en charge sa conception s'est basée sur des jeux ou manipulations déjà expérimentées dans d'autres outils itinérants ou animations bien connues dans le réseau des clubs d'astronomie de la région. 
Le plateau et les accessoires de jeu.

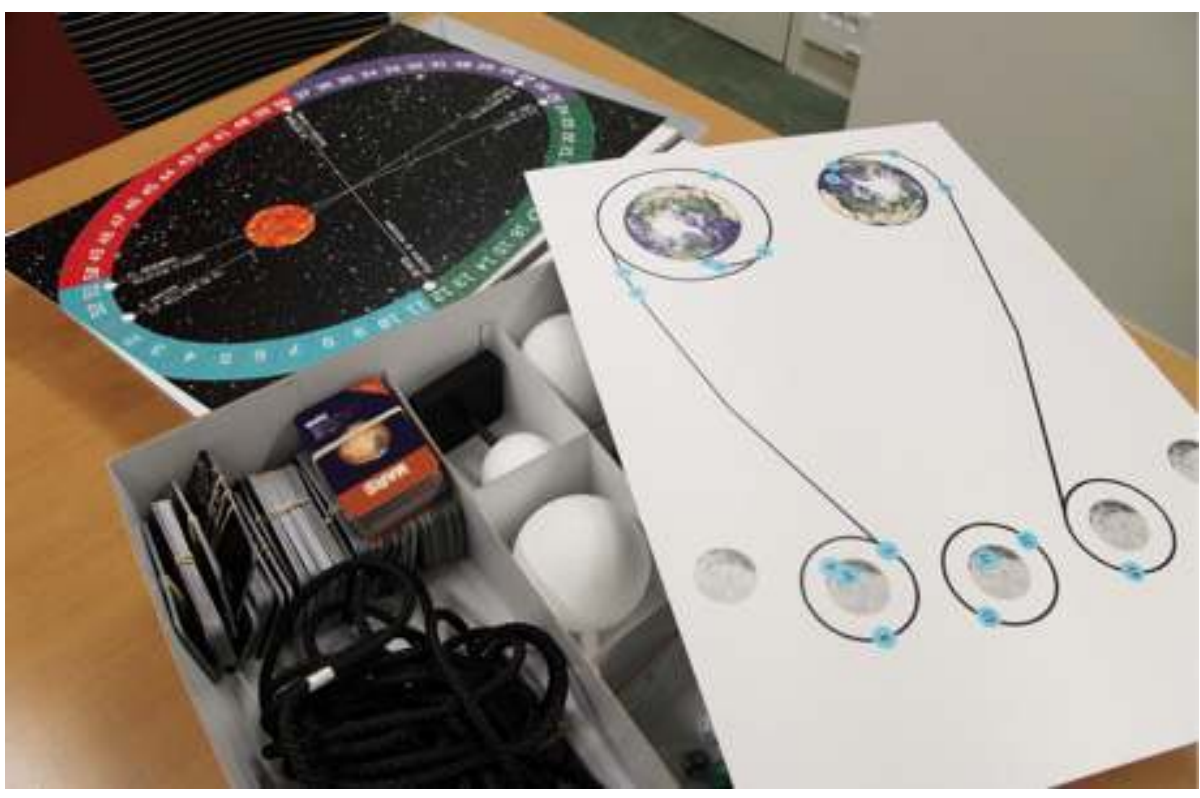

(c) Forum des Sciences/S. Lericque

15 Le kit d'animation se présente sous la forme d'une boîte en carton de 60 x 40 centimètres de dimensions, contenant l'ensemble des éléments pouvant assurer les différentes séances : boules en polystyrène, maquettes de la Lune, plateaux de jeux, mètre enrouleur, corde, jeux de cartes, pions et dés... Le kit baptisé À la découverte du ciel est composé d'ateliers qui permettent de développer de manière progressive le thème de l'astronomie à travers un parcours éducatif. Les animations sont indépendantes mais les thèmes abordés répondent à une certaine logique astronomique, grosso modo, du plus proche (le ciel vu de la Terre) au plus lointain (l'Univers à grande échelle). Pour un cycle de plusieurs séances, il est préférable de suivre l'ordre des animations. Le cycle d'animation se décline ainsi :

16 - «Réalise ton ciel » : les enfants sont invités à dessiner sur un fond de carte étoilée les constellations figurant des personnages, des animaux ou des objets liés à la mythologie ;

17 - «Les constellations 3D» : les enfants positionnent dans une salle des sphères à des endroits a priori aléatoires, pour finalement se rendre compte que celles-ci dessinent des constellations bien connues;

18 - «Les étoiles» : par l'intermédiaire d'un jeu de cartes, les enfants prennent conscience que les étoiles ne sont pas toutes identiques et qu'elles sont surtout différentes des planètes ;

19 - «L'ordre des planètes » : les enfants doivent imaginer une phrase mnémotechnique pour retenir l'ordre des planètes du Système solaire ;

20 - « Les planètes » : grâce à un jeu de cartes, les enfants prennent conscience qu'il n'y a pas que les planètes dans le Système solaire, mais aussi tout un tas d'autres corps : astéroïdes, comètes, satellites ;

21 - «Les cycles de la Terre » : par un jeu de dés, les enfants font connaissance avec les deux principaux mouvements de la Terre que sont la rotation et la révolution et appréhendent les notions de jour, de saison ou d'année ; 
22 la Lune ;

24 différentes échelles de l'Univers;

25 - «Représenter la Terre » : les enfants doivent imaginer un message à envoyer vers une éventuelle civilisation extraterrestre destiné à leur présenter notre petite planète Terre.

Le livret d'animation du jeu.

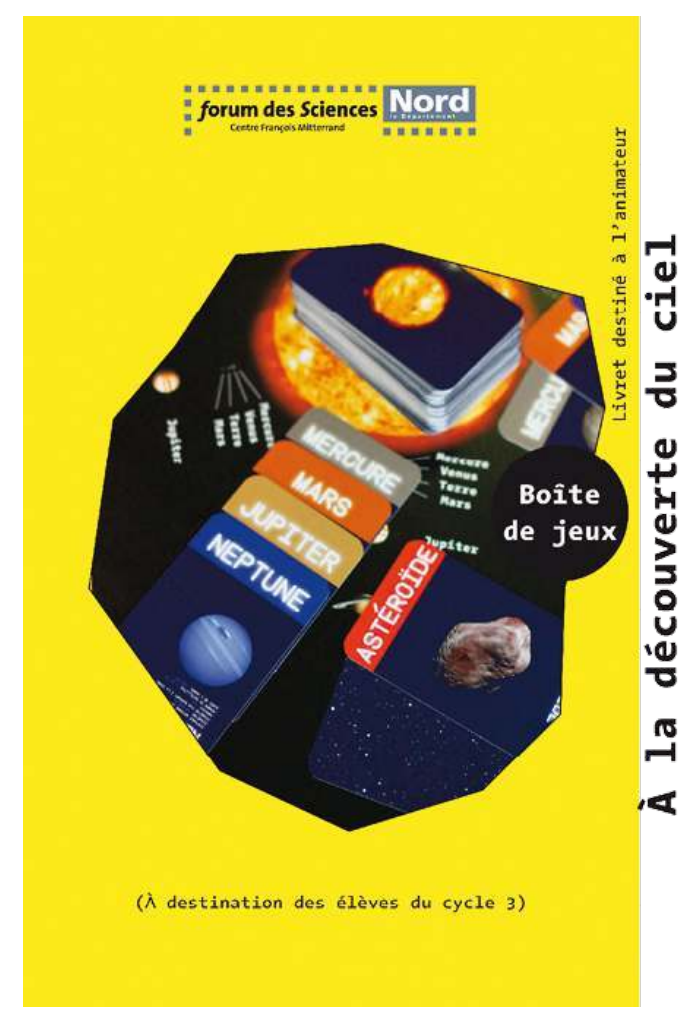

(C) Forum des Sciences/S. Lericque

Un livret d'animation d'une quarantaine de pages vient compléter l'ensemble des éléments matériels. C'est d'ailleurs, au sens de l'équipe de conception, l'élément le plus important... Ce livret propose un scénario d'animation pour chaque thématique et rappelle pour chacune des séances les mots clés et les notions importantes abordées. Ces scenarii sont évidemment adaptables par n'importe quel animateur.

Un DVD est également livré avec la boîte. On trouve dans ce dernier plusieurs éléments qui permettent le bon déroulement de certaines animations mais aussi bon nombre de diaporamas, de vidéos, de documents, permettant à l'animateur d'aller plus loin ou de répondre, avec l'appui de visuels, aux questions posées par les enfants.

L'outil comprend également une formation technique et théorique donnée par un médiateur astronome. En l'occurrence, dans le présent cas, les animateurs recrutés par la Ville de Lille, ont suivi deux journées de formation : une première approche sur les 
grands principes astronomiques - les mouvements de la Terre, la Lune, le Système solaire, les étoiles, l'Univers à grande échelle - et ont pu ensuite prendre en main la boîte et ses différents jeux.

\section{Et après ?}

Les premiers retours d'expérience sont plutôt positifs. Même si la fragilité de la boîte et la contrainte qu'elle soit utilisée pour un public trop jeune sont parfois soulignées par les animateurs, les enfants qui participent aux animations, ainsi que leurs parents sont plutôt enthousiastes. Certains enseignants se basent même sur le contenu des animations pour appuyer leurs cours. Malgré tout, le Forum départemental des Sciences reste attentif aux retours d'expériences des animateurs de la Ville de Lille et fait son possible - en fonction des contraintes économiques et matérielles - pour répondre à leurs attentes. Ainsi, plusieurs améliorations ont d'ores et déjà été appliquées dans une seconde version de la boîte À la découverte du ciel.

Forts de cette première expérience, le Forum départemental des Sciences et la Ville de Lille ont déjà décidé de poursuivre leur partenariat, toujours dans le cadre des activités périscolaires. Ainsi, deux nouvelles boîtes de jeux verront le jour ces prochains mois. La première aura toujours pour thème l'astronomie - et le monde qui nous entoure au sens large - mais sera adaptée aux enfants du cycle 2. Enfin, une échéance plus lointaine prévoit la sortie d'une autre boîte ayant pour thématique l'électricité.

Décrivez-nous votre fonction?

Témoignage de Béatrice Sauvage, intervenante thématique sciences à la Ville de Lille

Décrivez-nous votre fonction?

Je suis animatrice intervenante sur la thématique sciences pour la Ville de Lille. J'interviens dans le cadre des NAP mises en place par la Ville de Lille à la rentrée 2014. J'anime aujourd'hui autour de deux outils, la boîte de jeux du Forum départemental des Sciences et les malles du musée d'Histoire naturelle de Lille. Je travaille avec des classes de grande section, de CP et CM sur des cycles de 12 séances d'1 $\mathrm{h} 30$. Chaque trimestre je change d'école. Les groupes sont en général des classes de $20 / 30$ enfants.

Comment avez-vous appréhendé l'animation en astronomie avec la boîte de jeux ?

Nous avons eu deux jours de formation au Forum, l'une sur les grands concepts en astronomie et l'autre sur l'utilisation de la boîte. Je suis physicienne de formation et j'avais quelques notions en astronomie, cela a facilité les choses mais à mon sens, le livret d'animation fourni et quelques recherches personnelles suffisent à acquérir les connaissances nécessaires pour animer l'outil.

Je suis intervenue auprès d'enfants de grande section alors que l'outil n'a pas été conçu pour eux initialement. J'ai du adapter mes interventions, simplifier les ateliers proposés. Le DVD fourni avec la boîte de jeux m'a aidé sur ce plan. La boîte propose 10 ateliers, or je travaille sur un cycle de 12 séances. En général, je fais une séance 
introductive au sujet, à partir d'un livre ou d'images issues de revues pour en concevoir un poster. Et la dernière séance est consacrée à une séance de planétarium du Forum. C'est le clou du spectacle pour les enfants.

Justement, quelles sont les réactions des enfants?

C'est très différent d'un groupe à l'autre, d'une école à l'autre. Mais tous sont très curieux. Les étoiles, le ciel les fascinent. Ils s'émerveillent des images astronomiques. Les ateliers de la boîte sont ludiques et les enfants apprécient. Certains thèmes comme les phases de la lune sont plus complexes à aborder notamment chez les plus jeunes.

Quelles sont les forces et les faiblesses de cet outil?

Je travaille avec d'autres outils du musée d'Histoire naturelle de Lille, je peux donc comparer les approches. Ce qui est très intéressant avec la boîte c'est qu'il y a un fil rouge, il y a une logique de sens. Les malles du musée d'Histoire naturelle, qui ont d'autres intérêts, sont utilisées séance par séance et n'ont pas de lien entre elles. L'autre atout de la boîte est sa praticité : petite et très légère, elle rassemble le matériel nécessaire et la logistique est faible. Par contre, il manque quelques ateliers pour permettre aux enfants de repartir à la maison avec un objet qu'ils ont fabriqué, or à la différence des malles du musée, la boîte de jeux n'en propose que très peu. Il serait nécessaire aussi de proposer plus de variantes, plus de suggestions d'animation dans le livret, notamment pour l'adapter à des plus jeunes ou à des enfants qui ont besoin de changer d'activités souvent.

\section{Le point de vue de la directrice du projet éducatif global à la Ville de Lille, Hélène Hannoir}

Comment avez-vous mis en place les nouvelles activités périscolaires au sein des écoles de la Ville?

Nous avons réfléchi dans le cadre horaire imparti (un créneau de $1 \mathrm{~h} 30$ ) à déployer des contenus éducatifs de qualité. Différents thèmes sont abordés, culturels, sportifs, scientifiques.

Tous ces thèmes suivent un fil rouge : solliciter le langage et l'enrichir.

Les NAP entrent dans le cadre du Projet éducatif global défini par la Ville, différents plans thématiques ont été définis qui consistent à offrir des contenus aux enseignants à partir des projets de classe. En 2013, nous avions fait le bilan qu'il manquait d'offres en science. Nous avons dans un premier temps travaillé avec le musée d'Histoire naturelle de la Ville de Lille puis grâce aux NAP, nous avons enrichi nos propositions avec le Forum départemental des Sciences et l'astronomie. La Ville de Lille a un engagement fort dans l'éducation. Pour les familles, les NAP sont gratuites.

Quels regards portent les enseignants sur les NAP mises en place?

C'est très variable. Certains estiment que ce sont deux temps différents - le temps de la classe et le temps des NAP - qui ne s'interpénètrent pas. D'autres, trouvent les 
contenus riches et s'en emparent en classe pour poursuivre l'enrichissement. Notre objectif est vraiment qu'il y ait des passerelles entre les deux, que les NAP servent aussi l'enseignant.

Quel premier bilan faites-vous?

Nous menons des évaluations. Les enfants fréquentent à $87 \%$ les NAP, un taux équivalent à la fréquentation en classe. Nous menons actuellement une évaluation auprès des enfants, les premiers retours sont très satisfaisants, les premiers indicateurs sont donc au vert.

Et l'année prochaine?

Nous sommes actuellement en train de finaliser les NAP pour la prochaine rentrée. Nous avons effectué des appels d'offre afin de trouver des prestataires associatifs. Le musée d'Histoire naturelle de Lille développe actuellement un plan « culture scientifique ». Nous collaborons avec l'INRIA sur le développement d'un thème sur la robotique et nous poursuivrons notre collaboration avec le Forum départemental des Sciences autour de deux thèmes, l'électricité et l'astronomie pour les plus jeunes.

\section{RÉSUMÉS}

Fort de son expérience de plus de 20 ans en matière de conception d'activités scientifiques à destination du jeune public, le Forum départemental des Sciences de Villeneuve d'Ascq a été sollicité pour créer un outil pédagogique adapté aux nouvelles activités périscolaires : les concepteurs retracent les étapes de réalisation de cet outil et dressent avec les utilisateurs un premier bilan.

\section{INDEX}

Mots-clés : Outil pédagogique, nouvelles activités périscolaires, forum départemental des sciences

\section{AUTEURS}

\section{COLETTE CAZIN}

responsable de la communication du Forum départemental des Sciences colette.cazin@cg59.fr

\section{SIMON LERICQUE}

médiateur scientifique au planétarium du Forum départemental des Sciences simon.lericque@cg59.fr 